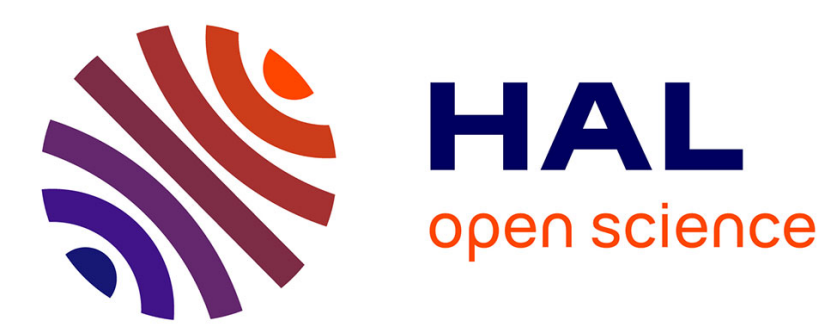

\title{
Simple Asymptotic BER Expressions for LoRa System over Rice and Rayleigh Channels
}

Vincent Savaux, Guillaume Ferré

\section{To cite this version:}

Vincent Savaux, Guillaume Ferré. Simple Asymptotic BER Expressions for LoRa System over Rice and Rayleigh Channels. Wireless Telecommunications Symposium, Apr 2021, San Francisco (virtual),

United States. hal-03200448

\section{HAL Id: hal-03200448 \\ https://hal.science/hal-03200448}

Submitted on 16 Apr 2021

HAL is a multi-disciplinary open access archive for the deposit and dissemination of scientific research documents, whether they are published or not. The documents may come from teaching and research institutions in France or abroad, or from public or private research centers.
L'archive ouverte pluridisciplinaire HAL, est destinée au dépôt et à la diffusion de documents scientifiques de niveau recherche, publiés ou non, émanant des établissements d'enseignement et de recherche français ou étrangers, des laboratoires publics ou privés. 
archives-ouvertes

\title{
Simple Asymptotic BER Expressions for LoRa System over Rice and Rayleigh Channels
}

\author{
Vincent Savaux, Guillaume Ferré
}

\section{To cite this version:}

Vincent Savaux, Guillaume Ferré. Simple Asymptotic BER Expressions for LoRa System over Rice and Rayleigh Channels. Wireless Telecommunications Symposium, Apr 2021, San Francisco, United

States. hal-03200448

\section{HAL Id: hal-03200448 \\ https://hal.archives-ouvertes.fr/hal-03200448}

Submitted on 16 Apr 2021

HAL is a multi-disciplinary open access archive for the deposit and dissemination of scientific research documents, whether they are published or not. The documents may come from teaching and research institutions in France or abroad, or from public or private research centers.
L'archive ouverte pluridisciplinaire HAL, est destinée au dépôt et à la diffusion de documents scientifiques de niveau recherche, publiés ou non, émanant des établissements d'enseignement et de recherche français ou étrangers, des laboratoires publics ou privés. 


\section{Simple Asymptotic BER Expressions for LoRa System over Rice and Rayleigh Channels}

\author{
Vincent Savaux \\ Advanced Connectivity Lab \\ $\mathrm{b}<>$ com, Rennes, France \\ Email: vincent.savaux@b-com.com
}

\author{
Guillaume Ferré \\ Univ. Bordeaux, CNRS, \\ Bordeaux INP, IMS, UMR 5218, \\ F-33400, Talence, France \\ Email: guillaume.ferre@enseirb-matmeca.fr
}

\begin{abstract}
This paper deals with the bit error rate (BER) of LoRa signal over Rayleigh and Rice channels. Exact analytical expressions of the BER in LoRa systems have been proposed in the literature, but they are not tractable and raise problems of computation precision. This is mainly due to the fact that these expressions involve sums of binomial coefficients, leading to extremely large numbers and leading to problem of precision. We then hereby suggest asymptotic expressions of the BER, which are at the same time tractable and accurate over a wide BER range. These expressions do not involve sums of binomial coefficients anymore, but only closed-form functions, and can then be easily obtained through computer simulations.
\end{abstract}

\section{INTRODUCTION}

Among the digital communication technologies used in the low power wide area network (LPWAN) world, LoRa is the most studied by the scientific $\mathrm{c}$ ommunity $\mathrm{t}$ o date. This is mainly due to the open deployment strategy chosen by Semtech. The digital communication technique used in LoRa modulation is the chirp spread spectrum (CSS) initially described by Winkler in [1]. Despite a high sensitivity to poor time/frequency synchronization, this technique offers many advantages such as i) a constant envelope which allows to operate with power amplifiers (PAs) in s aturation m ode and thus to have excellent amplification efficiency, ii) a sensitivity that can be optimized at the cost of a loss of spectral efficiency, iii) a low complexity demodulation based on fast Fourier transform (FFT).

LoRa or more generally CSS has given rise to numerous studies and analyses in recent years. At the physical layer level, one will retain works proposing to optimize the receivers in order to improve the synchronization steps or to find solutions to destructive collisions [2]. However, in order to be able to judge the effectiveness of the proposed processing, it is necessary to situate the performance in terms of bit error rate (BER) of the developed algorithms based on the theoretical results. Thus [3] and [4], [5] have proposed approximates and closed expressions of the binary error probabilities of LoRa system over additive white Gaussian noise (AWGN) channels. However, LoRa propagation channels are generally non frequency-selective for which only few literature proposes a closed form expression of binary error probability. In [4], expressions of the BER of LoRa signal over Rayleigh channel
TABLE I

OVERVIEW OF BER ANALYSIS OF LORA SYSTEM ACCORDING TO DIFFERENT MODELS AND APPROACHES.

\begin{tabular}{|l|l|l|}
\hline Reference & Channel & Approach \\
\hline$[3]-[5]$ & AWGN & $\begin{array}{l}\text { exact [5] } \\
\text { approximation [4] } \\
\text { Monte-Carlo [3] }\end{array}$ \\
\hline$[8]$ & AWGN+channel coding & approximation \\
\hline$[7]$ & AWGN+interference & approximation \\
\hline$[6]$ & Rayleigh, Rice, Nakagami & exact \\
\hline
\end{tabular}

have been suggested, but they are approximations based on error functions. In [6], the exact expression of the BER for LoRa signal over different channel models has been derived. However, despite their exactness, the BER expressions in [6] are not tractable, and lead to problem of precision. This is mainly due to the fact that the solutions involve sums of binomial coefficients whose full precision computation requires hundreds of bits.

We provide in Table I a summary of the literature dealing with BER derivation in LoRa system according to the channel model (AWGN, Rayleigh, etc.) and the approach (exact or approximated BER expression). Note that we have added [7] and [8] to enlarge the scope of this overview. In fact, these paper does not deal with BER of LoRa signal over specific channel model, but their BER analyses consider the effect of the channel coding and the impact of interference on the BER performance, respectively.

In this paper we derive a very simple and tractable asymptotic expression of the BER of LoRa signal over Rice and Rayleigh channels from that developed in [6], which tends to the exact BER for low noise variance value. It is shown through simulations that this approximation matches well the exact BER behaviors [6] in wide BER ranges. Furthermore, the suggested solution only involves straightforward functions. Thus, the proposed BER expressions is a good trade-off between accuracy and simplicity, allowing for a fast assessment of the achievable performance of a LoRa signal over Rice and Rayleigh channels. Moreover, we provide a summary of the literature dealing with more sophisticated BER expressions in different channel models, as well as including the effect of interference [7] and channel coding [8].

The rest of the paper is organized as follows: Section II 
is dedicated to the description of the LoRa modulation and demodulation principles and to the introduction of the exact BER expression. The derivation of a very simple approximation of theses closed form expressions is developed in Section III. The simulations results are presented in Section IV, and we draw conclusions in Section V.

\section{BACKGROUND}

LoRa uses several spreading factors $(S F)$ to control the bit rate, improve the range, and decrease the energy consumption. Initially, the binary information flow generated from the medium access control (MAC) layer is divided into subsequences, each of length $S F \in[7 \ldots 12]$. The set of $S F$ consecutive bits constitutes a symbol. The number of possible symbols is hence equal to $M=2^{S F}$. In the LoRa context, $S F$ also indicates the spreading factor and the relation between the bit rate $D_{b}$ and the symbol rate $D_{s}$ that can be written as: $D_{s}=D_{b} / S F$. Spread spectrum is obtained through a signal known as chirp that varies continuously and linearly in frequency. When the derivative of the frequency variation is positive, then we deal with an up chirp, conversely it is a down chirp.

When the chirp is up or down over the entire symbol period $T_{s}$, which is also called the signaling interval, it is identified as raw chirp. Its complex envelope is mathematically expressed as follows, for $t \in\left[-\frac{T_{s}}{2}, \frac{T_{s}}{2}\right)$ :

$$
c(t)=e^{j \theta_{c}(t)},
$$

with $\theta_{c}(t)= \pm \pi \frac{B}{T_{s}} t^{2}$ where $B$ is the frequency excursion of the transmitted chirp. The ' + ' and '-' signs stand for up and down raw chirp, respectively. If we note $f_{c}(t)$ the chirp frequency variation in time, we have:

$$
f_{c}(t)=\frac{1}{2 \pi} \frac{d \theta_{c}(t)}{d t}= \pm \frac{B}{T_{s}} t .
$$

For digital communication systems with no spreading spectrum, the bandwidth used by the transmitted signal is proportional to the symbol rate. The coefficient of proportionality depends on the shaping filter, which is in general a half Nyquist one. In the CSS modulation used in LoRa, the signal bandwidth is fixed by $B$ which has the following relationship with $T_{s}$ :

$$
M=B \times T_{s} .
$$

To distinguish between the $M$ different symbols of the constellation, $M$ orthogonal chirps have to be defined so that each symbol exhibits a specific instantaneous phase trajectory. In the sequel, we denote $m_{i}$ the transmitted symbol at time $i T_{s}$, with $m_{i} \in\{0, \ldots, M-1\}$ and $i \in \mathbb{N}$. The chirp associated to the $i$-th transmitted symbol is then obtained from the raw chirp by applying a delay $\tau_{i}=\frac{m_{i}}{B}$ and the raw chirp outside the interval $\left[-\frac{T_{s}}{2}, \frac{T_{s}}{2}\right)$ is cyclically brought back into $\left[-\frac{T_{s}}{2},-\frac{T_{s}}{2}+\tau_{i}\right]$. As a consequence, the the instantaneous phase modulated chirp (e.g. up chirp) related to the transmission of the symbol $m_{i}$ breaks down into two parts:

$$
\begin{aligned}
\theta_{c}^{i}(t)= & \pi\left[\frac{B}{T_{s}}\left(t-2 \tau_{i}\right) t+2 B t\right] \\
& \text { for } t \in\left[-\frac{T_{s}}{2},-\frac{T_{s}}{2}+\frac{m_{i}}{B}\right) \\
\theta_{c}^{i}(t)= & \frac{\pi B}{T_{s}}\left(t-2 \tau_{i}\right) t \\
& \text { for } t \in\left[-\frac{T_{s}}{2}+\frac{m_{i}}{B}, \frac{T_{s}}{2}\right) .
\end{aligned}
$$

Accordingly, the complex envelope of the LoRa symbol transmitting the symbol $m_{i}$ is $s_{i}(t)=e^{j \theta_{c}^{i}\left(t-i T_{s}\right)}$.

In the following, we consider perfect time and frequency synchronizations and a one tap block fading channel $h \sim$ $\mathcal{N}_{\mathbb{C}}\left(\mu, \sigma_{h}^{2}\right)$. Thus, $|h|$ obeys a Rice distribution if $\mu \neq 0$, and a Rayleigh distribution if $\mu=0$. Moreover, we introduce the Rice coefficient $\kappa=\frac{\lambda}{\sigma_{h}^{2}}$, where $\lambda=|\mu|^{2}$. Under these assumptions, the received signal sampled at rate $T_{e}$ and denoted by $y_{i}\left(n T_{e}\right)$ can be written as

$$
y_{i}\left(n T_{e}\right)=h \times s_{i}\left(n T_{e}\right)+w\left(n T_{e}\right),
$$

where $w\left(n T_{e}\right) \sim \mathcal{N}_{\mathbb{C}}\left(0, \sigma^{2}\right)$ represents the complex noise assumed to be white, Gaussian and circular. Furthermore, $n$ is the sample index with $n \in \llbracket-\frac{N}{2}, \frac{N}{2}-1 \rrbracket, N$ being the number of samples of a LoRa symbol. At Nyquist rate, we have $T_{e}=\frac{1}{B}$, and then $M=N$.

At the receiver, the transmitted symbols are detected by multiplying every received signal $y_{i}$ by the conjugate version of the raw chirp (1) used in the transmitter, i.e. $r_{i}\left(n T_{e}\right)=$ $y_{i}\left(n T_{e}\right) e^{-j \theta_{c}\left(n T_{e}\right)}$. Then, when a non coherent demodulation strategy is used, the optimal estimation of $m_{i}$ can be performed by searching for the maximum of the periodogram of $r_{i}\left(n T_{e}\right)$, computed as $\mathcal{P}_{i}[k]=\left|R_{i}[k]\right|^{2}$, where $R_{i}[k]$, for $k \in \llbracket 0, N-$ $1 \rrbracket$, is the discrete Fourier Transform (DFT) of $N$ samples from $r_{i}\left(n T_{e}\right)$. Thus, the estimation of $m_{i}$ can be achieved by computing:

$$
\hat{m}_{i}=N-\underset{k \in \llbracket 0, N-1 \rrbracket}{\operatorname{argmax}}\left(\mathcal{P}_{i}[k]\right) .
$$

The symbol error probability, denoted by $P_{s}$, is defined as the probability that $\hat{m}_{i}$ is not equal to the actual transmitted symbol $m_{i}$, i.e. $P_{s}=\mathbb{P}\left[\hat{m}_{i} \neq m_{i}\right]$. The BER is then obtained through:

$$
P_{b}=\frac{2^{S F-1}}{2^{S F}-1} P_{s} .
$$

It has been proved in [6] that, in the absence of prior information, all the symbols can be considered as equiprobable so that the symbol error probability over a Rice channel, denoted by $P_{s}^{\text {Rice }}$, is expressed as

$$
\begin{aligned}
P_{s}^{\text {Rice }}= & -\sum_{k=1}^{N-1} \frac{\left(\begin{array}{c}
N-1 \\
k
\end{array}\right)(-1)^{k} \sigma^{2}}{k N \sigma_{h}^{2}+(k+1) \sigma^{2}} \\
& \times \exp \left(-\frac{\lambda k N}{k N \sigma_{h}^{2}+(k+1) \sigma^{2}}\right) .
\end{aligned}
$$


It must be noticed that the error probability over Rayleigh channel (denoted by $P_{s}^{R a y}$ in the following) is straightforwardly deduced from (8) by setting $\lambda=0$. Alternatively, the symbol error probability in AWGN channel is obtained by setting $\left(\lambda=1, \sigma_{h}^{2}=0\right)$. In any case, despite the exactness of the expression (8), it requires the computation of the high-valued binomial coefficient $\left(\begin{array}{c}N-1 \\ k\end{array}\right)$. This may suffer from precision problems since we remind that, in LoRa we have $N \in\{128,256, . ., 4096\}$. It results that (8) is hardly computable in practice nor tractable, therefore limiting its application to theoretical results. This is the reason why we hereby provide simple expressions of $P_{s}^{\text {Rice }}$ and $P_{s}^{\text {Ray }}$ in asymptotic cases where the noise variance value is low.

\section{ASYMPTOTIC BER EXPRESSION}

In this section we provide simple expressions of the asymptotic BER value for LoRa system over Rice and Rayleigh channels.

Proposition. Let the noise variance value $\sigma^{2}$ be low enough to consider that $k \geq 1>>\frac{1}{N \cdot \frac{\sigma_{h}^{2}}{\sigma^{2}}+1}$, and $k N \sigma_{h}^{2}>>(k+$ 1) $\sigma^{2}$. Then, for any $N=2^{S F}$, we can make the following approximation

$$
P_{s}^{\text {Rice }} \approx e^{-\kappa} \times \frac{\gamma+\ln (N-1)}{N \cdot \frac{\sigma_{h}^{2}}{\sigma^{2}}+1},
$$

where $\gamma$ is the Euler-Mascheroni constant $\gamma \approx 0.577$.

Proof. According to the assumption of low $\sigma^{2}$ value, then, for any $k=0,1, . ., N-1$, the exponential functions in (8) can be approximated as

$$
\exp \left(-\frac{\lambda k N}{k N \sigma_{h}^{2}+(k+1) \sigma^{2}}\right) \approx \exp (-\kappa) .
$$

The substitution of (10) into (8) then leads to

$$
P_{s}^{\text {Rice }} \approx-e^{-\kappa} \sum_{k=1}^{N-1} \frac{\left(\begin{array}{c}
N-1 \\
k
\end{array}\right)(-1)^{k} \sigma^{2}}{k N \sigma_{h}^{2}+(k+1) \sigma^{2}} .
$$

Then, in conditions where $1>>\frac{1}{N \cdot \frac{\sigma_{h}^{2}}{\sigma^{2}}+1}, P_{s}^{\text {Rice }}$ in (11) can be simplified as

$$
P_{s}^{\text {Rice }} \approx-\frac{e^{-\kappa}}{N \cdot \frac{\sigma_{h}^{2}}{\sigma^{2}}+1} \sum_{k=1}^{N-1} \frac{\left(\begin{array}{c}
N-1 \\
k
\end{array}\right)(-1)^{k}}{k} .
$$

Moreover, we consider $N$ large enough to assume that the approximation $\left(\begin{array}{c}N-1 \\ k\end{array}\right) \underset{N \rightarrow+\infty}{\sim} \frac{(N-1)^{k}}{k !}$ holds, which yields

$$
P_{s}^{\text {Rice }} \approx-\frac{e^{-\kappa}}{N \cdot \frac{\sigma_{h}^{2}}{\sigma^{2}}+1} \sum_{k=1}^{N-1} \frac{(N-1)^{k}}{k \cdot k !} .
$$

Then, let

$$
E_{1}(z)=\int_{z}^{+\infty} \frac{e^{-t}}{t} d t
$$

with $z \in \mathbb{C} \backslash \mathbb{R}^{-}$, be the exponential integral function, defined in [9]. The series representation of $E_{1}$ is

$$
E_{1}(z)=-\gamma-\ln (z)-\sum_{k=1}^{+\infty} \frac{(-1)^{k} z^{k}}{k k !} .
$$

Since $\lim _{z \rightarrow+\infty} E_{1}(z)=0$, and reminding that we assume $N \rightarrow$ $+\infty$, then the substitution of $z$ by $(N-1)$ in the sum of (14) and then in (13) leads to (9), which concludes the proof.

The asymptotic error probability in Rayleigh channel is straightforwardly obtained when $\kappa=0$, leading to

$$
P_{s}^{R a y} \approx \frac{\gamma+\ln (N-1)}{N \cdot \frac{\sigma_{h}^{2}}{\sigma^{2}}+1} .
$$

It should be noted that (9) and (15) are more tractable expressions than (8) as they only involve usual functions. Furthermore, the suggested approximations do not require the computation of $\left(\begin{array}{c}N-1 \\ k\end{array}\right)$, which leads to problems of computation precision, especially when $N$ is large (e.g. $N=$ $1024,2048,4096)$. In the following, we investigate through simulations the accuracy and the domain of validity of our approximations of the error probability.

\section{Simulations Results}

Simulations have been performed to verify the accuracy of the suggested approximations of the error probability in (9) and (15). Theoretical results have been obtained using Python 3.6 with scipy and gmpy2 packages for mathematical computing and precision improvement, respectively ${ }^{1}$. It must be emphasized that the theoretical results presented in [6] had been validated through simulations. As a consequence, we do not reproduce the Monte-Carlo simulations to avoid excessive plots.

Fig. 1 shows the BER performance of LoRa system over Rayleigh and Rice channel models versus $1 / \sigma^{2}$ (in $\mathrm{dB}$ ) for SF7 (a) and SF12 (b). Other series of simulations have been undertaken for SF8 to SF11, but the results are not shown in this paper, as the BER performance is equivalent to that of SF7, except that the BER curves are shifted to the left (a shift of about $-3 \mathrm{~dB}$ per $\mathrm{SF}$ ). The performance of the exact expression (8) obtained from [6] (referred as "exact") is compared with that of suggested approximations (9) and (15) (referred as "approx.") for $\kappa=0$ (Rayleigh), $\kappa=1$, and $\kappa=4$.

It can be observed in Figs. 1-(a) and (b) that the larger the Rice coefficient $\kappa$, the lower the BER. This is a very wellknown result since asymptotically, the case $\kappa=+\infty$ corresponds to the AWGN channel, which is a lower performance bound. Otherwise, we notice that the domain of validity of the suggested approximations depends on the coefficient $\kappa$. In fact, we can see that in the Rayleigh case $(\kappa=0)$, the asymptotic expression well matches the exact performance for BER values lower than $10^{-1}$, which is relevant as a BER range larger than $10^{-1}$ is usually not of interest in practice. For the

\footnotetext{
${ }^{1}$ The code can be found at https://github.com/b-com/ber_LoRa
} 


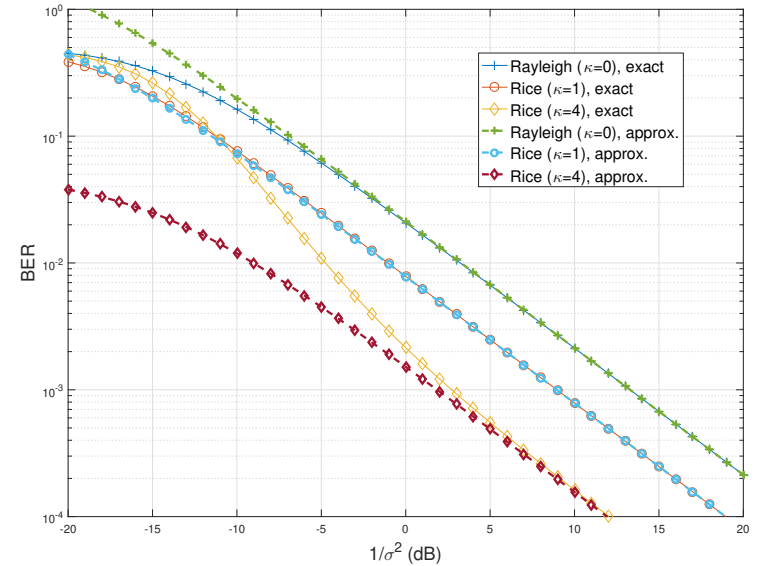

(a) SF7.

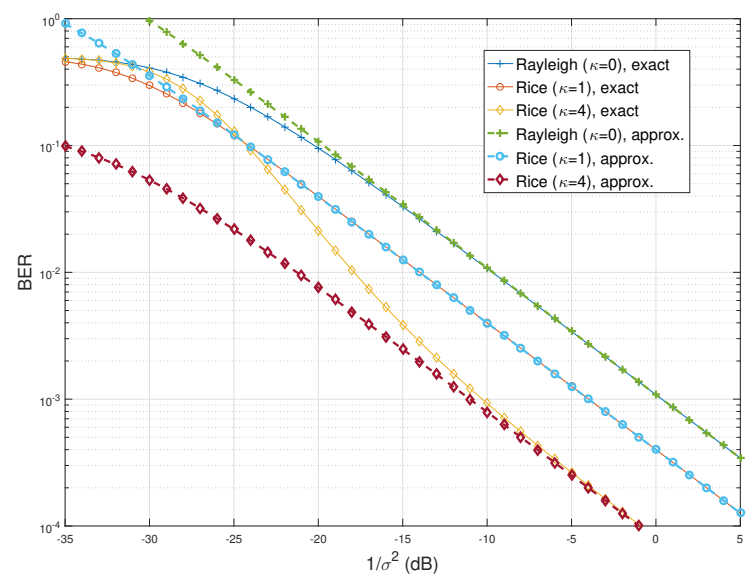

(b) SF12.

Fig. 1. BER versus $1 / \sigma^{2}(\mathrm{~dB})$ for SF7 (a) and SF12 (b), comparison of the exact BER expression (8), and the suggested approximations (9) and (15) for $\kappa=0$ (Rayleigh), $\kappa=1$, and $\kappa=4$.

Rice channel with $\kappa=1$, we observe that the approximation well matches the exact expression for any BER value, and for $\kappa=4$, the asymptotic expression of the BER well matches the exact one for BER values lower than $10^{-3}$. We deduce that the suggested approximations are more relevant for lower values of the Rice coefficient $\kappa$, which is consistent with theory since no asymptotic BER expression can be derived for the AWGN channel corresponding to $\kappa=+\infty$ [5].

The accuracy of the suggested BER expressions compared with the exact one is very interesting in practice, especially since (8) is not tractable, and raises some computing precision problems. In fact, to give an idea of the required precision, the full precision computation of the binomial coefficient $\left(\begin{array}{c}N-1 \\ k\end{array}\right)$ in (8) in SF7 requires at least 150 bits, and even up to 4000 bits in SF12 (e.g. $\left(\begin{array}{c}4096 \\ 200\end{array}\right)$ returns infinity in Matlab), which can be achieved only with the use of the package gmpy2 in python. This practically shows the relevance of the suggested asymptotic BER expressions, in addition to their accuracy.

\section{Conclusion}

In this paper, we have derived simple asymptotic expressions of the BER of LoRa signal over Rice and Rayleigh channel, considering low noise variance value. We have shown through simulations the validity of the suggested approximated expressions compared with the exact one. The advantage of the proposed analysis is to obtain tractable and easily computable solutions, whereas the computation of the exact BER expression may raise problems of precision, or requires large variables sizes. In general, the suggested expressions well fit the exact BER value over a wide BER range. Moreover, an overview of the available literature dealing with BER analysis in LoRa system according to different models and approaches has been provided as well.

\section{REFERENCES}

[1] M. Winkler, "Chirp signals for communications," IEEE WESCON Convention Record, p. 7, 1962.

[2] B. Laporte-Fauret, M. A. Ben Temim, G. Ferré, D. Dallet, B. Minger, and L. Fuché, "An enhanced lora-like receiver for the simultaneous reception of two interfering signals," in 2019 IEEE 30th Annual International Symposium on Personal, Indoor and Mobile Radio Communications (PIMRC), 2019, pp. 1-6.

[3] G. Ferré and A. Giremus, "LoRa Physical Layer Principle and Performance Analysis," in proc. of IEEE International Conference on Electronics, Circuits and Systems 2018 (ICECS'18). Bordeaux, France: IEEE, 12 2018, pp. $1-4$.

[4] T. Elshabrawy and J. Robert, "Closed-Form Approximation of LoRa Modulation BER Performance," IEEE Communications Letters, vol. 22, no. 9 , pp. 1778 - 1781, September 2018.

[5] J. Proakis and M. Salehi, Digital Communications. McGraw-Hill, 2008.

[6] C. F. Dias, E. R. de Lima, and G. Fraidenraich, "Bit Error Rate ClosedForm Expressions for LoRa Systems under Nakagami and Rice Fading Channels," Sensors, vol. 19, no. 20, pp. 1 - 11, October 2019.

[7] O. Afisiadis, M. Cotting, A. Burg, and A. Balatsoukas-Stimming, "On the Error Rate of the LoRa Modulation with Interference," IEEE Transactions on Wireless Communications, vol. 19, no. 2, pp. 1292 - 1304, February 2020.

[8] M. J. Faber, K. M. van der Zwaag, W. G. V. dos Santos, H. R. d. O. Rocha, M. E. V. Segatto, and J. A. L. Silva, "A Theoretical and Experimental Evaluation on the Performance of LoRa Technology," IEEE Sensors Journal, vol. 20, no. 16, pp. 9480-9489, August 2020.

[9] M. Abramowitz and I. Stegun, Handbook of Mathematical Functions with Formulas, Graphs, and Mathematical Tables. Dover, 1970, ch. 5: Exponential Integral and Related Functions, pp. 227 - 237. 